\title{
Posibles riesgos para la salud debido al consumo de aspartame
}

\section{(Possible health risks due to the consumption of aspartame)}

\author{
Teresa Guerrero Villegas ${ }^{1}$, Galo Mora Flores ${ }^{1}$
}

\begin{abstract}
Resumen:
Los edulcorantes son compuestos que confieren el sabor dulce a alimentos, bebidas y medicamentos. El aspartame es uno de los más utilizados en la actualidad; su metabolismo produce fenilalanina, ácido aspártico y metanol. El propósito de esta investigación fue revisar la literatura científica sobre los niveles de consumo permitidos, toxicología y estudios epidemiológicos del aspartame. La Directiva del Parlamento Europeo lo aprobó como aditivo alimentario en 1994 y la FDA, en 1996. El Comité Mixto FAO/OMS de Expertos en Aditivos Alimentarios y la FDA fijaron la ingestión diaria admisible en 40 y $50 \mathrm{mg} / \mathrm{kg}$ de peso corporal/ día, respectivamente. El edulcorante y sus productos de degradación han sido evaluados por más de 30 años con la intervención de numerosas organizaciones internacionales. Sin embargo, aún existe controversia sobre su uso porque hay investigaciones cuyos resultados le atribuyen efectos secundarios neuropsíquicos, tumores cerebrales, propiedades cancerígenas para diferentes órganos, daños para el feto durante la gestación, desarrollo de linfomas y leucemias, mientras que otros investigadores aseguran que su uso es inocuo para los seres humanos si el consumo es menor que la ingestión diaria admisible. No es recomendado para personas con fenilcetonuria y mujeres embarazadas.
\end{abstract}

Palabras clave: Aspartame; ingestión diaria admisible; carcinogénesis; fenilcetonuria.

\begin{abstract}
:
Sweeteners are compounds that give the sweet taste to foods, drinks and drugs. Aspartame is one of the most used today; its metabolism produces phenylalanine, aspartic acid and methanol. The purpose of this research was to review the scientific literature about the levels of consumption considered safe, toxicology and epidemiological data of aspartame. The European Parliament approved it as a food additive in 1994 and the FDA did it in 1996. Joint FAO/WHO Expert Committee on Food Additives and FDA set the Acceptable Daily Intake at 40 and $50 \mathrm{mg} /$ $\mathrm{kg}$ bw/day, respectively. The sweetener and its degradation products have been evaluated for over 30 years with the involvement of numerous international organizations. However, there is still controversy over its use because there are researches whose results attribute to it neuropsychiatric side effects, brain tumors, carcinogenic properties for different organs, damage to the fetus during pregnancy, development of lymphomas and leukemia, while other researchers say their use is harmless to humans if consumption is less than the Acceptable Daily Intake. It is not recommended for people with phenylketonuria and pregnant women.
\end{abstract}

Keywords: Aspartame; acceptable daily intake; carcinogenesis; phenylketonuria.

\footnotetext{
${ }^{1}$ Universidad Tecnológica Equinoccial, Facultad de Ciencias de la Ingeniería, Quito - Ecuador
} (gvet2640@ute.edu.ec,gmora@ute.edu.ec) 


\section{Introducción}

Pese a que, a nivel mundial, la dieta de los seres humanos comprende una amplia variedad de productos animales, vegetales y minerales, desde tiempos ancestrales la humanidad ha tenido una marcada preferencia hacia los alimentos dulces (Durán et al., 2011), lo que explica que a lo largo de la historia se hayan obtenido y sintetizado compuestos que satisfagan esa necesidad.

El primer edulcorante del que se tiene registro es la miel, utilizada en las antiguas culturas de Grecia (aprox. 500 - 300 AC) y China (3000 - 220 AC). En las cuevas de la Araña ${ }^{2}$, en España, se encontró pinturas rupestres que datan de más de 20 mil años; entre ellas, la denominada "El hombre de Bicorp", que representa a un hombre subiendo por lianas para obtener miel de abejas (Cuevas de la Araña, s.f.; Weihrauch \& Diehl, 2004).

La miel fue reemplazada más tarde por la sacarosa o azúcar común, originalmente obtenida de la caña de azúcar. Durante las Guerras Mundiales, la remolacha azucarera era la principal fuente de sacarosa (Weihrauch \& Diehl, 2004).

A fines del siglo XVII la comunidad médica ya responsabilizó a la ingesta de azúcar de provocar muchas enfermedades, y ante esta situación surgió la necesidad de buscar sustitutos que proporcionen las mismas cualidades y sensaciones que produce el azúcar, pero sin afectar a la salud (Durán, et al., 2011). Sin embargo, el primer edulcorante artificial, la sacarina, fue sintetizado recién en 1879 por Remsen y Fahlberg (Weihrauch \& Diehl, 2004); ésta es aproximadamente 300 veces más dulce que la sacarosa, pero tiene un regusto amargo, sobre todo a altas concentraciones (Weininger \& Stermitz, 1988).

Astiasarán y Martínez (2003) definen edulcorante como "toda sustancia capaz de proporcionar sabor dulce al alimento que la contiene" y agrupan a estos en tres categorías:

i) Edulcorantes naturales

- Monosacáridos: glucosa, fructosa, galactosa

- Disacáridos: sacarosa, lactosa, maltosa

ii) Edulcorantes nutritivos, derivados de productos naturales

- Productos que provienen del almidón: glucosa, jarabe de glucosa, isoglucosa

- Productos que provienen de la sacarosa: azúcar invertido

- Azúcares-alcoholes o polioles: sorbitol, manitol, xilitol, maltitol

${ }^{2}$ Las cuevas de la Araña son un conjunto de cuevas epipaleolíticas cerca del río Escalona en Bicorp, municipio de la Comunidad Valenciana de España. 
- Neoazúcares: fructo-oligosacáridos

iii) Edulcorantes intensos

- Edulcorantes de síntesis o artificiales: aspartame, acesulfamo, sacarina, ciclamato, alitamo, dulcina

- Edulcorantes intensos de origen vegetal: taumatina, esteviósido, monelina, dihidrocalcona, glicirrizina

\section{Aspartame}

Es un edulcorante artificial intenso, bajo en calorías (EFSA, 2013). Fue descubierto en 1965 por James Schlatter, quien realizaba una investigación con aminoácidos para desarrollar un tratamiento contra las úlceras y derramó por accidente un poco de sustancia sobre su mano; cuando se lamió el dedo, se dio cuenta de que tenía un sabor dulce. Schlatter trabajaba para la multinacional farmacéutica G.D. Searle and Company, que fue la primera propietaria de la marca comercial del aspartame: NutraSweet®. En 1985 la empresa química Monsanto adquirió G.D. Searle and Company y en el año 2000 J.W. Childs Equity Partners compró la compañía NutraSweet de Monsanto (The NutraSweet Company, s.f.).

La comercialización del aspartame en Estados Unidos fue autorizada por primera vez en 1974. Pocos meses después, la autorización fue suspendida debido a que los primeros estudios no habían evaluado correctamente su toxicidad y carcinogenicidad para el cerebro. Tras nuevas valoraciones y examen de los datos, la FDA (siglas que corresponden a U.S. Food and Drug Administration) autorizó su comercialización en alimentos sólidos en 1981, y en bebidas refrescantes en 1983. En 1996 fue autorizado como edulcorante general (The NutraSweet Company, s.f.).

Desde su introducción al mercado en 1981 como NutraSweet® millones de personas en todo el mundo han consumido aspartame en más de 6000 productos que contienen este endulzante; entre ellos: gomas de mascar, edulcorantes artificiales de mesa, agua saborizada, gelatinas, gaseosas y otros productos dietéticos, zumos en polvo, yogures, cereales, medicamentos pediátricos y salsas para cocinar (Magnuson et al., 2007; The NutraSweet Company, s.f.).

La Directiva del Parlamento Europeo aprobó el aspartame como aditivo alimentario el 30 de junio de 1994 y su uso está permitido en más de 90 países (Aspartame, s.f.; EFSA, 2013).

\subsection{Identificación, propiedades y síntesis}

El número CAS (Chemical Abstracts Service) del aspartame es 22839-47-0, que corresponde a una identificación numérica única asignada por la Sociedad Americana de 
Química (CAS, 2014). Su número E (código para los aditivos alimentarios en la Unión Europea, UE) es E 951 (EFSA, 2013).

El nombre IUPAC (siglas que corresponden a International Union of Pure and Applied Chemistry) es: L-alfa-aspartil-L-fenilalanina-1-metil éster (FDA, 2013).

El peso molecular del aspartame es 294,3 daltons. Su fórmula molecular es $\mathrm{C}_{14} \mathrm{H}_{18} \mathrm{~N}_{2} \mathrm{O}_{5}$ y su fórmula estructural se muestra en la Figura 1.

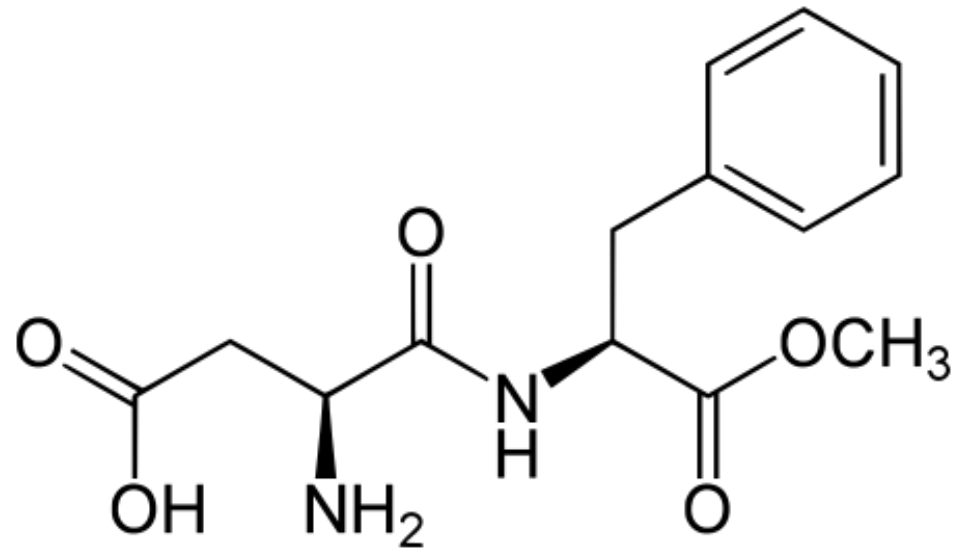

Figura 1. Aspartame

Fuente: Wikimedia Commons [Public domain]

El aspartame es un éster metílico de un dipéptido formado por fenilalanina y ácido aspártico. Es un sólido blanco, cristalino, sin olor, aproximadamente 200 veces más dulce que la sacarosa o azúcar común y aporta 4 calorías por gramo (Durán et al., 2011).

El punto isoeléctrico del aspartame es 5,2; a medida que se aleja de este $\mathrm{pH}$ por acidificación o alcalinización del medio, su solubilidad en agua aumenta. Para muchos usos se prefiere sus sales hidrosolubles. El aspartame no se solubiliza en aceites y grasas (Cubero, Montferrer \& Villalta, 2002).

Es estable cuando se encuentra seco o congelado, pero se descompone y pierde su poder edulcorante con el transcurso del tiempo cuando se conserva en líquidos a temperaturas superiores a $30^{\circ} \mathrm{C}$, por lo que no debería ser utilizado en alimentos cocinados o esterilizados. La descomposición del aspartame produce dicetopiperazina, que no posee propiedades edulcorantes, y por hidrólisis se transforma en metanol, ácido aspártico y fenilalanina (Cubero et al., 2002; EFSA, 2013).

Actualmente se encapsula el aspartame con aceites hidrogenados para que sea liberado al final del calentamiento, con lo cual puede ser utilizado en productos horneados. También se lo mezcla con otros edulcorantes, pues así aumenta su estabilidad frente a procesos que requieren calor (Cubero et al., 2002; Gianuzzi \& Molina, 1995). 
El aspartame se sintetiza a partir de fenilalanina y ácido aspártico, por vía química o por vía enzimática. La síntesis química genera productos secundarios y su principal impureza es la dicetopiperazina (Aspartamo, s.f.; Cubero, et al., 2002).

\subsection{Ingestión diaria admisible}

El Codex Alimentarius (2014) define la ingestión diaria admisible (IDA) como "la ingestión diaria que, durante una vida, parece no conllevar riesgos aparentes para la salud de los consumidores... Se expresa en miligramos de producto químico por kilogramo de peso corporal."

La IDA del aspartame para los seres humanos se fijó en $40 \mathrm{mg} / \mathrm{kg}$ de peso corporal/día por el JECFA (Comité Mixto FAO/OMS de Expertos en Aditivos Alimentarios) en 1981 (FAO, 2014); en Estados Unidos, la FDA la ha fijado en $0-50 \mathrm{mg} / \mathrm{kg}$ de peso corporal/día (International Sweeteners Association, s.f.; Magnuson et al., 2007).

\subsection{Metabolismo}

Tras su ingestión, el aspartame se metaboliza en el tracto intestinal, por acción de esterasas y peptidasas, en fenilalanina, ácido aspártico y metanol, de modo que casi nada de aspartame llega a la sangre. La fenilalanina es un aminoácido esencial (que el organismo humano no puede sintetizar por sí mismo). El ácido aspártico es uno de los veinte aminoácidos con los que las células forman las proteínas, pero no es un aminoácido esencial; es importante en la síntesis de ADN y como neurotransmisor. El metanol se encuentra, en pequeñas cantidades, de forma natural en el organismo y en un gran número de alimentos (Cubero et al., 2002).

En el cuerpo, el metabolismo del ácido aspártico y del metanol producidos es rápido, por lo que no se eleva significativamente la concentración de estas dos sustancias en la sangre, aun si se consumiera en una sola toma una cantidad equivalente a la IDA (Aspartamo, s.f.; International Sweeteners Association, s.f.).

Si el consumo supera la IDA, el nivel de fenilalanina en sangre puede aumentar proporcionalmente con la dosis administrada. Sin embargo, estos valores no son importantes, excepto para personas que presentan fenilcetonuria (PKU) (Aspartamo, s.f.).

La PKU es una afección congénita causada por la carencia de la enzima fenilalanina hidroxilasa, lo que impide descomponer apropiadamente la fenilalanina. Consecuentemente, este aminoácido se acumula y resulta tóxico para el sistema nervioso central, ocasionando daño cerebral (MedlinePlus, 2013). Por esta razón, en la UE y otros países los productos alimenticios que contienen aspartame deben indicarlo en la etiqueta, con su nombre o su número $\mathrm{E}$ (EFSA, 2013). 


\subsection{Controversia sobre el consumo de aspartame}

El aspartame y sus productos de degradación han sido evaluados por más de 30 años por medio de estudios experimentales en animales, investigación clínica, estudios epidemiológicos y vigilancia post-comercialización, con la intervención de numerosas organizaciones, incluyendo el JECFA y el Comité Científico de la Comisión Europea sobre la Alimentación Humana (SCF) (EFSA, 2013).

La FDA y la EFSA han aprobado al aspartame como aditivo alimentario en base a investigaciones que arrojan resultados positivos sobre su inocuidad. Sin embargo, aún persiste la polémica entre ciertos sectores, debido a que también hay numerosos reportes que alertan sobre su toxicidad.

A continuación se citan algunas investigaciones realizadas al respecto:

Según Gianuzzi y Molina (1995), el aspartame presenta efectos secundarios neuropsíquicos, como dolor de cabeza, insomnio, irritabilidad, depresión, fatiga, vértigo, problemas visuales, gastrointestinales y menstruales. Cuando se consume en dosis elevadas puede provocar inhibición del supresor del apetito.

Olney et al. (1996) analizaron los datos de tumores cerebrales en Estados Unidos durante dos décadas y encontraron que se incrementaron en dos ocasiones: el primer incremento, que fue leve, se asoció a un mejoramiento en la tecnología de diagnóstico, y un segundo, más reciente y sostenido, implicó al aspartame debido a un estudio que reveló una alta incidencia de tumores cerebrales en ratas alimentadas con el edulcorante, en comparación con la ausencia de estos tumores en los controles. Los autores concluyeron que hay necesidad de volver a evaluar el potencial carcinogénico del aspartame.

Luego de investigar el efecto de los metabolitos del aspartame, Schwartz escribió una carta al Diario Western de Medicina que fue publicada en 1999, en la que sugirió una relación entre el aspartame y el aumento de cáncer de mama y de próstata. Sostuvo que el aspartame es parcialmente metabolizado a metanol, que a su vez se convierte en formaldehído; así se acumula dentro de las células e induce el cáncer. En el mismo número de la revista, el Dr. Trichopoulos (cuyas investigaciones eran financiadas por Monsanto) respondió a la carta explicando que el aumento de la tasa de cáncer de mama ocurrió antes de la introducción del aspartame, y ha ido decreciendo durante los años siguientes (Schwartz, 1999).

Por otro lado, Soares, Azoubel y Batigália (2007) administraron aspartame en una concentración de $14 \mathrm{mg} / \mathrm{kg}$ a un grupo de ratas preñadas, y agua a otro grupo que actuó como control. En el grupo que recibió aspartame hubo reducción en los pesos promedio de 
la placenta y materno-fetal, en el largo del cordón umbilical y en la mayoría de los parámetros cariométricos del núcleo de los hepatocitos. Se concluyó que su uso durante la gestación puede ser perjudicial para el feto.

Weihrauch y Diehl (2004) investigaron artículos escritos sobre edulcorantes artificiales. Fue de su interés la publicación de Olney et al. (1996), sobre la que dijeron no ser admisible la correlación entre el aumento de los tumores cerebrales y la introducción del aspartame, por tratarse de una "falacia ecológica", además de que sus resultados no pudieron ser confirmados por ensayos posteriores.

Las conclusiones de Weihrauch y Diehl fueron las siguientes:

- El "uso pesado" de edulcorantes artificiales (mayor a 1,7 g por día) conduce a un aumento del riesgo relativo de 1,3 para el cáncer de vejiga en humanos. No es posible determinar los agentes exactos, debido a que muchos edulcorantes artificiales se combinan en los productos alimenticios.

- A pesar de los artículos no científicos publicados y de las publicaciones científicas, no hay evidencia de que el aspartame sea cancerígeno (Weihrauch \& Diehl, 2004).

El Centro de Investigación del Cáncer Cesare Maltoni de la Fundación Europea Ramazzini, con sede en la ciudad italiana de Bolonia, llevó a cabo un ensayo en el que se administró aspartame a 1800 ratas, en dosis diarias de 100 000, 50000, 10000, 2000, 400, 80 y 0 ppm. El tratamiento duró hasta la muerte de los animales, y a todos se les sometió a necropsia completa. Tras la evaluación histopatológica de órganos y tejidos los investigadores publicaron los siguientes resultados:

a) Aumento de la incidencia de tumores malignos, en particular en las hembras tratadas a 50000 ppm.

b) Aumento de linfomas y leucemias, particularmente en las hembras tratadas con dosis de $100000,50000,10000,2000$ y 400 ppm.

c) Aumento de la incidencia de carcinoma (tumor maligno) de células de transición de la pelvis renal y del uréter en hembras tratadas con dosis de $100000,50000,10000,2000$ y 400 ppm.

d) Aumento de la incidencia de schwannomas malignos (tumores de la vaina de mielina) de los nervios periféricos con una tendencia positiva en los machos.

Se concluyó que el aspartame es un agente cancerígeno multipotencial, incluso a una dosis diaria de $20 \mathrm{mg} / \mathrm{kg}$ de peso corporal, que es menor que la IDA. También se sugirió una urgente reevaluación de las directrices sobre su uso y consumo (Soffritti et al., 2006). 
Luego de los primeros resultados del Instituto Ramazzini, Magnuson et al. (2007) realizaron una revisión crítica de literatura científica sobre el aspartame y concluyeron:

- Los niveles de uso actuales del aspartame, incluso por altos usuarios se mantienen muy por debajo de la IDA fijada por la EFSA y por la FDA.

- El consumo de grandes cantidades de aspartame en una dosis única puede tener efecto sobre algunos parámetros bioquímicos, incluyendo los niveles de aminoácidos en plasma y los niveles de neurotransmisores del cerebro.

- Los estudios de toxicidad del aspartame y sus productos de descomposición, realizados en ratones, ratas, hámsteres y perros han encontrado efectos adversos del edulcorante con dosis desde $4000 \mathrm{mg} / \mathrm{kg}$ de peso corporal/día.

- Los estudios de carcinogenicidad llevados a cabo sobre el aspartame no cuentan con evidencias aceptables de que la sustancia es cancerígena.

- La hipótesis de que el aspartame afecta la función del sistema nervioso, de aprendizaje o comportamiento no se ve apoyada por los resultados de las investigaciones.

Los investigadores de la Fundación Ramazzini volvieron a publicar sus resultados ampliados y concluyeron que el gran número de animales utilizados y la larga duración de la investigación fueron factores determinantes para que se haya revelado la carcinogenicidad del aspartame, pues ellos observaron un aumento de linfomas y leucemias en las hembras, y de tumores sólidos como tumores malignos de los nervios periféricos, del cráneo y de la pelvis renal en ratas, además de tumores de hígado en ratones. Consideraron su trabajo prioritario para la protección de la salud pública, en particular la salud de los niños y las mujeres embarazadas que se encuentran entre las poblaciones vulnerables, tomando en cuenta el uso cada vez mayor de edulcorantes artificiales, no solo aspartame, sino otras mezclas utilizadas en miles de alimentos (Belpoggi et al., 2006).

Todos los estudios realizados en el Instituto Ramazzini fueron evaluados por la EFSA y por la FDA y desechados argumentando numerosos errores metodológicos, manteniendo que el aspartame es seguro para el consumo humano (EFSA, 2013; FDA, 2007).

En junio de 2011 se informó que científicos del Instituto Ramazzini fueron recibidos por parlamentarios europeos con respecto a los resultados obtenidos en sus investigaciones sobre el aspartame. A raíz de esto, los parlamentarios consiguieron que la Comisión Europea solicite a la EFSA que comience un nuevo proceso de reevaluación de este compuesto en 2012 (Navarro, 2014). 
En el sitio Web de la EFSA se encuentran publicados, desde Diciembre de 2013 los resultados y conclusiones de estas reevaluaciones:

- El aspartame se hidroliza rápida y completamente a fenilalanina, ácido aspártico y metanol en el tracto gastrointestinal.

- A partir de estudios de toxicidad crónica en animales se identificó un NOAEL (Nivel sin efecto adverso observable) de $4000 \mathrm{mg} / \mathrm{kg}$ de peso corporal/día. No se excluye la posibilidad de que se presente toxicidad en animales a dosis inferiores a $4000 \mathrm{mg} / \mathrm{kg}$.

- Se atribuye el desarrollo de toxicidad en animales a la fenilalanina, pues se conoce que este aminoácido a niveles plasmáticos elevados causa toxicidad para los seres humanos.

- Se concluyó que el aspartame no constituye un problema de seguridad si su ingesta es menor a la IDA de $40 \mathrm{mg} / \mathrm{kg}$ de peso corporal/día. Por lo tanto, no hay razón para revisar la IDA del aspartame. Sin embargo, esta IDA no es aplicable a pacientes con PKU.

El 13 de diciembre de 2013 el Instituto Ramazzini publicó un comunicado de la Directora del Centro de Investigación del Cáncer Cesare Maltoni en respuesta al informe de la EFSA sobre el aspartame. En él, Fiorella Belpoggi escribe:

"Seguimos convencidos de que el aspartame es un carcinógeno para los seres humanos, debido a que en nuestro modelo experimental ha demostrado ser cancerígeno en dos especies animales, la rata y el ratón, y para diversos órganos diana..."

La Dra. Belpoggi argumenta haber seguido los criterios de la Agencia Internacional para la Investigación sobre el Cáncer de la Organización Mundial de la Salud para clasificar los agentes carcinógenos. Manifiesta también que la EFSA no consideró otros estudios epidemiológicos importantes, como el de Schernhammer et al. (2012) que incluye 122000 personas y relaciona el uso de aspartame con un aumento en la incidencia de linfomas y leucemias, ni el de Andreatta et al. (2008), llevado a cabo en Argentina, que evidenció un incremento de tumores del tracto urinario en animales de experimentación tratados con aspartame (Belpoggi, 2013).

También indica la Dra. Belpoggi que próximamente contarán con la colaboración del NIEHS/ USA (Instituto Nacional de Ciencias de la Salud Ambiental de los Estados Unidos) con la finalidad de eliminar las incertidumbres sobre los datos obtenidos en sus experimentos sobre el aspartame; se realizará una reevaluación desde el punto de vista estadístico y se procederá a la caracterización de lesiones clasificadas como linfomas pulmonares, y finalmente en 2015 se llamará a un Grupo de Trabajo Internacional de Patología para la revisión de todos los tumores malignos diagnosticados (Belpoggi, 2013). 


\section{Conclusiones y Recomendaciones}

Pese a las investigaciones que alertan sobre el consumo de aspartame, según la FDA y la ESFA, ni el edulcorante ni sus metabolitos representan un riesgo para la salud de los seres humanos, si su ingesta es menor que la IDA, fijada en 50 y $40 \mathrm{mg} / \mathrm{kg}$ de peso corporal/día, respectivamente.

En vista de que las investigaciones sobre la seguridad del consumo de aspartame arrojan resultados contradictorios, sería importante conocer el informe definitivo de la reevaluación de su carcinogenicidad por parte del Instituto Ramazzini y el NIEHS/USA, que seguramente se publicará en el sitio Web del Instituto.

El aspartame, así como cualquier fuente alimenticia de fenilalanina no debe ser consumido por individuos con fenilcetonuria. Es recomendable que también se abstengan de su consumo mujeres embarazadas.

\section{Bibliografía}

Andreatta, M. M., Muñoz, S. E., Lantieri, M. J., Eynard, A. R. \& Navarro, A. (2008). Artificial sweetener consumption and urinary tract tumors in Cordoba, Argentina. Prev Med. 47(1):136-9. doi: 10.1016/j.ypmed.2008.03.015. Epub 2008 Apr 8.

Aspartamo. (s.f.). Recuperado de http://www.greenfacts.org/es/aspartamo/l-3/aspartame1.htm\#1p0

Astiasarán, I. \& Martínez, A. (2003). Alimentos. Composición y Propiedades. México: McGraw-Hill.

Belpoggi, F. (Diciembre 13, 2013). Aspartame: la risposta dell'Istituto Ramazzini alle dichiarazioni dell'EFSA. Recuperado del sitio Web del Instituto Ramazzini: http://www.ramazzini.org/news/aspartame-la-risposta-dellistituto-ramazzini-alledichiarazioni-dellefsa/

Belpoggi, F., Soffritti, M., Padovani, M., Degli Esposti, D., Lauriola, M. \& Minardi, F. (2006). Ann. N.Y. Acad. Sci. 1076: 559-577. doi: 10.1196/annals.1371.0

CAS, Chemical Abstracts Service. (2014). Recuperado de http://www.commonchemistry.org/ChemicalDetail.aspx?ref=22839-470\&terms=aspartame

Codex Alimentarius, Normas internacionales de los alimentos. (Febrero 1, 2014). Glosario de términos. Recuperado de http://www.codexalimentarius.org/pestres/Glossary/es 
Cubero, N., Montferrer, A. \& Villalta, J. (2002). Aditivos Alimentarios. Madrid: A. Madrid Vicente, Ediciones.

Cuevas de la Araña. (s.f.). Recuperado de http://www.rutasconhistoria.es/loc/cuevas-de-laarana

Durán, S., Quijada, M., Silva, L., Almonacid, N., Berlanga, M. \& Rodríguez, M. (2011). Niveles de ingesta diaria de edulcorantes no nutritivos en escolares de la región de Valparaíso [Versión electrónica], Rev. Chil. Nutr. 38(4), 444-449. Recuperado de http://www.scielo.cl/scielo.php?pid=S0717-75182011000400007\&script=sci_arttext

EFSA, European Food Safety Authority. (Diciembre 10, 2013). Aspartame. Recuperado de http://www.efsa.europa.eu/en/topics/topic/aspartame.htm

EFSA Panel on Food Additives and Nutrient Sources added to Food (ANS). Scientific opinion on the re-evaluation of aspartame $(E$ 951) as a food additive. EFSA Journal 2013;11(12):3496. Recuperado de http://www.efsa.europa.eu/en/efsajournal/doc/3496.pdf

FAO, Organización de las Naciones Unidas para la Alimentación y la Agricultura. (2014). Recuperado de http://www.fao.org/search/es/?cx=018170620143701104933\%3Apvqiwqrhhhq\&q=asp artame $\& x=4 \& y=5 \& \operatorname{cof}=F O R I D \% 3 A 9$

FDA, U.S. Food and Drug Administration. (Abril 20, 2007). FDA Statement on European Aspartame Study. Recuperado de http://www.fda.gov/food/ingredientspackaginglabeling/foodadditivesingredients/ucm20 8580.htm

FDA, U.S. Food and Drug Administration. (2013). Recuperado de http://www.accessdata.fda.gov/scripts/cdrh/cfdocs/cfcfr/cfrsearch.cfm?fr=172.804

Giannuzzi, L. \& Molina Ortiz. S. (1995). Edulcorantes Naturales y Sintéticos: Aplicaciones y Aspectos Toxicológicos. Acta Farnz. Botlaeretise 14 (2): 119-3 1. Recuperado de http://www.latamjpharm.org/trabajos/14/2/LAJOP_14_2_2_1_O7PY4U1EJI.pdf

International Sweeteners Association. (s.f.). Aspartamo. Recuperado de http://www.infoedulcorants.org/pdf/e951_aspartamo_sp.pdf

Magnuson, B. A., Burdock, G. A., Doull, J., Kroes, R. M., Marsh, G. M., Pariza, M. W., Spencer, P. S., Waddell, W. J. Walker, R. \& Williams, G. M. (2007). Aspartame: A Safety Evaluation Based on Current Use Levels, Regulations, and Toxicological and 
Epidemiological Studies. Critical Reviews in Toxicology, 37:629-727. DOI: $10.1080 / 10408440701516184$

MedlinePlus. (Septiembre 06, 2013). Fenilcetonuria. Recuperado de http://www.nlm.nih.gov/medlineplus/spanish/ency/article/001166.htm

Navarro, R. (Febrero, 25 del 2014). La EFSA descarta riesgos para la salud en el aspartamo. Malagahoy.es. Recuperado de http://www.malagahoy.es/article/salud/1667187/la/efsa/descarta/riesgos/para/salud/e spartamo.html

Olney, J. W., Farber, N. B., Spitznagel, E. \& Robins, L. N. (1996). Increasing brain tumor rates: is there a link to aspartame? J Neuropathol Exp Neurol, 55(11): 115-23. Recuperado de http://journals.Iww.com/jneuropath/Abstract/1996/11000/Increasing_Brain_Tumor_Rat es_Is_There_a_Link_to.2.aspx

Schernhammer, E. S., Bertrand, K. A., Birmann, B. M., Sampson, L., Willett, W. C. \& Feskanich, D. (2012). Consumption of artificial sweetener- and sugar-containing soda and risk of lymphoma and leukemia in men and women. Am J Clin Nutr, vol. 96 no. 6:1419-1428. DOI: 10.3945/ajcn.111.030833

Schwartz, G. R. (1999). Aspartame and breast and other cancers. West J Med, 171(56):300-301. Recuperado de http://www.ncbi.nlm.nih.gov/pmc/articles/PMC1308737/

Soares Pórtela, G., Azoubel, R. \& Batigália, F. (2007). Effects of Aspartame on MaternalFetal and Placental Weights, Length of Umbilical Cord and Fetal Liver: A Kariometric Experimental Study. Int. J. Morphol., 25(3):549-554. Recuperado de http://www.scielo.cl/scielo.php?script=sci_arttext\&pid=S0717$95022007000300012 \& \operatorname{lng}=$ es\&tlng=en. $10.4067 /$ S0717-95022007000300012.

Soffritti, M., Belpoggi, F., Degli Esposti, D., Lambertini, L., Tibaldi, E. \& Rigano, A. (2006). First Experimental Demonstration of the Multipotential Carcinogenic Effects of Aspartame Administered in the Feed to Sprague-Dawley Rats. Environ Health Perspect 114:379-385. DOI:10.1289/ehp.8711 available via http://dx.doi.org/ [Online 17 November 2005]

The NutraSweet Company. (s.f.). Recuperado de http://www.nutrasweet.com/company.asp Weihrauch, M. \& Diehl, V. (2004). Artificial sweeteners-do they bear a carcinogenic risk? Annals of Oncology, 15, 1460-1465. Recuperado de http://annonc.oxfordjournals.org/content/15/10/1460.full.pdf+html 
Weininger, S. \& Stermitz, F. (1988). Química Orgánica. Barcelona: Editorial Reverté, p. 836. Recuperado de http://books.google.com.ec/books?id=O6YvtgAtXmcC\&pg=PA836\&lpg=PA836\&dq=d ulzura+relativa+de+la+sacarina\&source=bl\&ots=xyjDLWuV8f\&sig=_3cMO6IWSjyTF1 mqHXKpLEnh8Bs\&hl=es\&sa=X\&ei=KcuCUsasKYqQkAeArICQDQ\&ved=0CDQQ6AE wAQ\#v=onepage\&q=dulzura\%20relativa\%20de\%20la\%20sacarina\&f=false 